\title{
Major cardiovascular and bleeding events with long-term use of aspirin in patients with prior cardiovascular diseases: 1-year follow-up results from the Management of Aspirin-induced Gastrointestinal Complications (MAGIC) study
}

\author{
Shinichiro Uchiyama ${ }^{1}\left(10\right.$. Shinya Goto ${ }^{2} \cdot$ Hideki Origasa $^{3} \cdot$ Naomi Uemura $^{4} \cdot$ Kentaro Sugano $^{5} \cdot$ Hideyuki Hiraishi $^{6,7}$. \\ Kazuyuki Shimada ${ }^{8} \cdot$ Yasushi Okada $^{9} \cdot$ Yasuo Ikeda $^{10}$ on behalf of The MAGIC Study Group
}

Received: 15 May 2019 / Accepted: 14 August 2019 / Published online: 24 August 2019

(C) The Author(s) 2019

\begin{abstract}
Aspirin should be used for the prevention of cardiovascular (CV) events by the risk-benefit balance. This study was conducted to clarify CV and bleeding events in Japanese aspirin users with a history of CV diseases. This study was a prospective, nationwide, multicenter cooperative registry of Japanese patients with CV diseases at risk of thromboembolism who were taking aspirin (75-325 mg) for at least 1 year. We observed major CV and bleeding events during follow-up. Patients with history of ischemic stroke (IS), transient ischemic attack (TIA), coronary artery disease (CAD), atrial fibrillation (AF), and venous thromboembolism (VTE) were included and analyzed in this sutdy. CV events included IS, TIA, CAD, CV death, angioplasty or stenting, and hospitalization because of CV disease. Bleeding events included major bleeding requiring hospitalization and/or blood transfusion. A total of 1506 patients were categorized into IS/TIA $(N=540)$, CAD $(N=632)$, and AF/VTE $(N=232)$. Among them, 101 patients had two or more categories. CV and bleeding events occurred in $61(3.82 \% /$ year) and 15 patients $(0.93 \%$ year), respectively. The annual rates of $\mathrm{CV}$ and bleeding events were $2.81 \%$ and $0.93 \%$ in IS/ TIA, $5.32 \%$ and $0.75 \%$ in CAD, $1.15 \%$ and $1.15 \%$ in $\mathrm{AF} / \mathrm{VTE}$, and $6.44 \%$ and $0.91 \%$ in two or more disease categories, respectively. The Management of Aspirin-induced Gastrointestinal Complications (MAGIC) study clarified the rates of major $\mathrm{CV}$ and bleeding events with long-term use of aspirin in patients with prior $\mathrm{CV}$ diseases in real-world clinical practice. The risk-benefit balance of aspirin was acceptable in patients with IS/TIA, CAD, and multiple CV diseases but not in those with AF/VTE.

Trial Registration: The MAGIC Study is registered at UMIN Clinical Trial Registry (www.umin.ac.jp/ctr/index-j.htm), number UMIN000000750.
\end{abstract}

Keywords Aspirin $\cdot$ Cardiovascular disease $\cdot$ Cardiovascular events $\cdot$ Hemorrhagic events $\cdot$ Registry

\section{Introduction}

Aspirin has been established to reduce the risk of cardiovascular (CV) events, including stroke, myocardial infarction (MI), and vascular death, in patients with a history of $\mathrm{CV}$ diseases [1, 2]. However, aspirin increases the risk of

A complete list of the MAGIC study group is provided in the Acknowledgements

Shinichiro Uchiyama

suchiyama@iuhw.ac.jp

Extended author information available on the last page of the article gastrointestinal (GI) injury, which may lead to GI bleeding, even at a low dose [3]. Therefore, the use of aspirin should be determined using the risk-benefit balance [4] Aspirin can be recommended in cases having a significant net clinical benefit estimated by reducing ischemic events and increasing hemorrhagic events.

The American Heart Association recommends the use of low-dose aspirin (75-325 mg) for patients having a 10-year CV event risk of $\geq 10 \%$ [5]. The US Preventive Services Task Force recommends prophylactic aspirin therapy only in patients with a 5-year CV risk $\geq 3 \%$ [6]. However, the risk of $\mathrm{CV}$ events is decreasing owing to the recent progress in risk factor management and may differ between ethnicities [7]. 
$\mathrm{CV}$ and hemorrhagic events were investigated during a 1-year follow-up period in Japanese patients receiving lowdose aspirin for the prevention of $\mathrm{CV}$ events enrolled in the Management of Aspirin-induced Gastrointestinal Complications (MAGIC) study.

\section{Methods}

\section{Study design}

The MAGIC study was a nationwide, multicenter-cooperative, prospective, observational study conducted in Japan. Details of the study design are described elsewhere [8]. Participants were enrolled from multiple disease categories, including those with coronary artery disease (CAD), ischemic stroke (IS), transient ischemic attack (TIA), atrial fibrillation (AF), and venous thromboembolism (VTE) requiring antithrombotic therapy, and recruited from 63 sites across the regions of Japan between April 2007 and September 2009. CV and hemorrhagic events as outcomes predefined in the protocol were evaluated by investigators at individual sites [8]. The study protocol was approved by the institutional review board at each site. All participants signed written informed consent forms. The study design was formulated by the Organization Committee (see "Acknowledgements": A complete list of the MAGIC study group), and data were collected through a Web-based system [8].

\section{Study population}

The inclusion and exclusion criteria were described elsewhere in detail [8]. Details of the background characteristics and risk factor profiles of the patients recruited at baseline are described elsewhere [9]. The incidence rates of major vascular and bleeding events in patients with $\mathrm{CV}$ diseases who were taking aspirin (75-325 mg daily) and followed up for 1 year were analyzed in this study.

Underlying CV diseases were categorized into IS/TIA, $\mathrm{CAD}$, and AF/VTE. CAD included myocardial infarction (MI) and angina pectoris.

The baseline data included sex, age, body height, body weight, body mass index, vascular risk factors including hypertension, dyslipidemia, diabetes mellitus, current cigarette smoking and alcohol drinking, use of aspirin (dosage and formulations: buffered or enteric coated), and concomitant use of other drugs.

\section{Outcomes}

$\mathrm{CV}$ events were judged by investigators in each site [8]. The incidence rates of $\mathrm{CV}$ events, including IS, TIA, CAD, CV death, coronary or other angioplasty or stenting, and hospitalization because of $\mathrm{CV}$ disease and bleeding events, or blood transfusion during the 1-year follow-up period were analyzed in this study.

\section{Statistical analysis}

The results were expressed as mean \pm standard deviation. The incidence rates and $95 \%$ confidence intervals (CIs) of bleeding and cardiovascular events were calculated using the Poisson method. All analyses were performed using R 2.15.1 (R Foundation for Statistical Computing, Department of Statistics and Mathematics, Wirtschafts University, Vienna, Austria). For all hypotheses, $p$ values $<0.05$ (twotailed) were considered significant.

\section{Results}

The baseline data on background characteristics, risk factors, and use of aspirin and other concomitant drugs are shown in Table 1. A total of 1506 patients were classified into the three disease categories, namely, IS/TIA $(N=540), \mathrm{CAD}(N=632)$, and AF/VTE $(N=232)$, on the basis of the underlying disease at baseline (Fig. 1). Among the patients, 61 had IS/TIA and CAD, 23 had IS/TIA and

Table 1 Characteristics of the patients at baseline

\begin{tabular}{ll}
\hline Characteristic & $\begin{array}{l}\text { Mean } \pm \text { SD } \\
\text { or number } \\
(\%)\end{array}$ \\
\hline Men (\%) & $1108(73.6 \%)$ \\
Age (year) & $68.2 \pm 9.5$ \\
Body height (cm) & $161.4 \pm 8.4$ \\
Body weight (kg) & $62.6 \pm 11.1$ \\
BMI & $23.9 \pm 3.2$ \\
Hypertension (\%) & $1077(71.5 \%)$ \\
Dyslipidemia (\%) & $848(56.3 \%)$ \\
Diabetes mellitus (\%) & $424(28.2 \%)$ \\
Current cigarette smoking (\%) & $158(10.5 \%)$ \\
Alcohol drinking (\%) & $606(40.2 \%)$ \\
Dose of aspirin (mg) & $103.9 \pm 26.0$ \\
Use of NSAIDs (\%) & $80(5.3 \%)$ \\
Use of other antiplatelet drugs (\%) & $368(24.4 \%)$ \\
Use of Warfarin (\%) & $179(11.9 \%)$ \\
Use of antihypertensive drugs (\%) & $1124(74.6 \%)$ \\
Use of lipid lowering drugs (\%) & $779(51.7 \%)$ \\
Use of antidiabetic drugs (\%) & $291(19.3 \%)$ \\
Use of antiarrhythmic drugs (\%) & $212(14.1 \%)$ \\
Use of antiulcer drugs (\%) & $785(52.1 \%)$ \\
\hline
\end{tabular}

$S D$ standard deviation, BMI body mass index, NSAIDs non-steroidal anti-inflammatory drugs 
Fig. 1 MAGIC population profile. IS/TIA ischemic stroke or transient ischemic attack, $C A D$ coronary artery disease, $A F /$

$V T E$ atrial fibrillation or venous thromboembolism

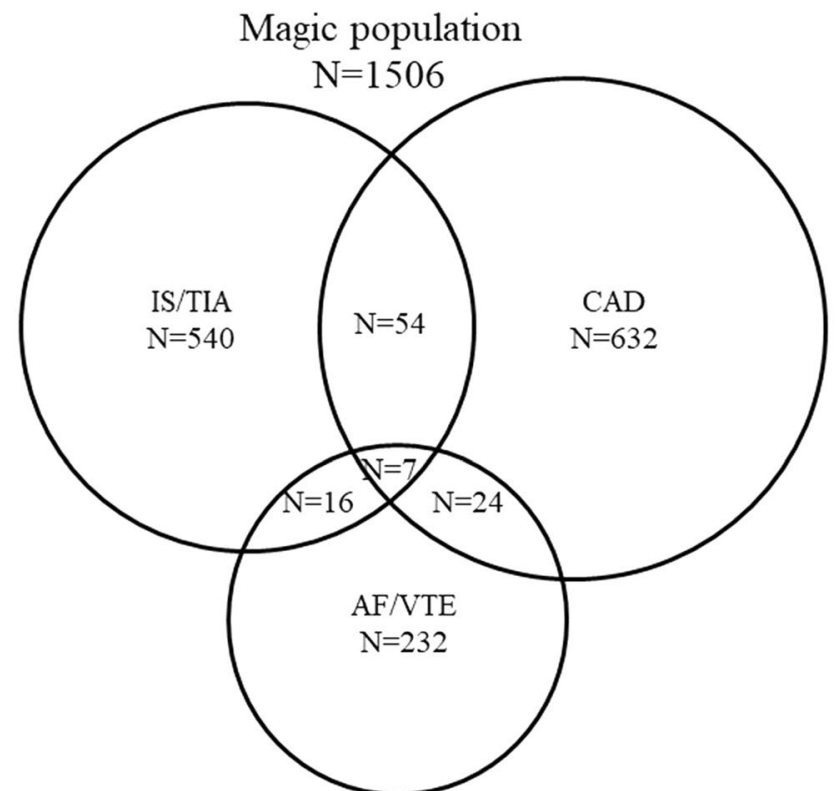

IS/TIA; ischemic stroke or transient ischemic attack, CAD; coronary artery disease, $\mathrm{AF} / \mathrm{VTE}$; atrial fibrillation or venous thromboembolism
Table 2 Major vascular and bleeding events

\begin{tabular}{lr}
\hline Major vascular and bleeding event & Number (\%/year, 95\% CI) \\
\hline Cerebrovascular or Cardiovascular event & $61(3.82 \%, 2.97-4.91 \%)$ \\
$\begin{array}{l}\text { Nonfatal stroke or transient ischemic } \\
\text { attack }\end{array}$ & $11(0.68 \%, 0.38-1.22 \%)$ \\
Nonfatal myocardial infarction & $5(0.31 \%, 0.13-0.73 \%)$ \\
Coronary angioplasty or stenting & $29(1.81 \%, 1.26-2.60 \%)$ \\
Hospitalization due to other vascular & $14(0.87 \%, 0.52-1.46)$ \\
$\quad$ event & $2(0.12 \%, 0.03-0.45 \%)$ \\
Cardiovascular death & $15(0.93 \%, 0.57-1.54 \%)$ \\
Major bleeding & $4(0.25 \%, 0.01-0.64 \%)$ \\
Cerebral bleeding & $10(0.62 \%, 0.34-1.14 \%)$ \\
Gastrointestinal bleeding & $1(0.06 \%, 0.01-0.35 \%)$ \\
Upper gastrointestinal bleeding & $9(0.56 \%, 0.29-1.06 \%)$ \\
Lower gastrointestinal bleeding & $1(0.06 \%, 0.01-0.35 \%)$ \\
Other bleeding &
\end{tabular}

AF/VTE, 31 had CAD and AF/VTE, and 7 had all of IS/ TIA, CAD and AF/VTE.

A total of 1506 patients were followed up for a mean of 390 days. $\mathrm{CV}$ events occurred in 61 patients $(3.82 \% /$ year; 95\% CI 2.97-4.91\%/year), and bleeding events occurred 15 patients (0.93\%/year; 95\% CI 0.57-1.54\%/ year) (Table 2). According to the disease categories at baseline, the annual incidence rates of $\mathrm{CV}$ and bleeding events were $2.81 \%$ and $0.93 \%$ in IS/TIA, $5.32 \%$ and $0.75 \%$ in CAD, $1.15 \%$ and $1.15 \%$ in AF/VTE, and $6.44 \%$ and $0.91 \%$ in two or more categories of diseases, respectively (Fig. 2).

\section{Discussion}

In our MAGIC study, the incidence rate of $\mathrm{CV}$ events was $3.82 \%$ /year and the incidence rate of bleeding events was $0.93 \% / y e a r$ (cerebral hemorrhage, $0.25 \%$ year and GI bleeding, $0.62 \% /$ year). According to a meta-analysis by the Antithrombotic Trialists' Collaboration (ATT), the annual incidence rate of vascular events, including MI, stroke, and vascular death, was $8.19 \%$ in the controls and $6.69 \%$ in patients who were receiving aspirin for secondary prevention of vascular disease [2]. The incidence rate of CV events in the MAGIC study was than that in the aspirin group of ATT. On the other hand, the incidence rates of bleeding events in the MAGIC study were comparable to those reported in the previous studies conducted in patients with a history of $\mathrm{CV}$ disease $[2,10,11]$.

In the Clopidogrel versus Aspirin in Patients at Risk of Ischaemic Events (CAPRIE) trial, the annual risk of stroke, MI, and vascular death was $5.83 \%$, whereas the annual risks of intracranial hemorrhage and GI bleeding were $0.47 \%$ and $0.72 \%$, respectively (any severe bleeding disorder was $1.55 \%$ ), in 9586 patients with ischemic stroke, MI, or peripheral vascular disease who were treated with aspirin $(325 \mathrm{mg} /$ day) [10]. In the Clopidogrel for High Atherothrombotic Risk and Ischemic Stabilization, Management and Avoidance (CHARISMA) trial, the rate of the primary efficacy end point, which was a composite of MI, stroke, and death from $\mathrm{CV}$ causes, was $7.3 \%$, and the incidence rate of severe bleeding was $1.3 \%$. The incidence rate of primary intracranial hemorrhage was $0.3 \%$, but the incidence rate of GI bleeding was not documented with aspirin (75-162 mg/day) alone in 
Fig. 2 Vascular and hemorrhagic events in 4 categories of patients. IS/TIA ischemic stroke or transient ischemic attack, $C A D$ coronary artery disease, $A F$ atrial fibrillation, $V T E$ venous thromboembolism, $C V E$ cardiovascular events, $M B I$ major bleeding events

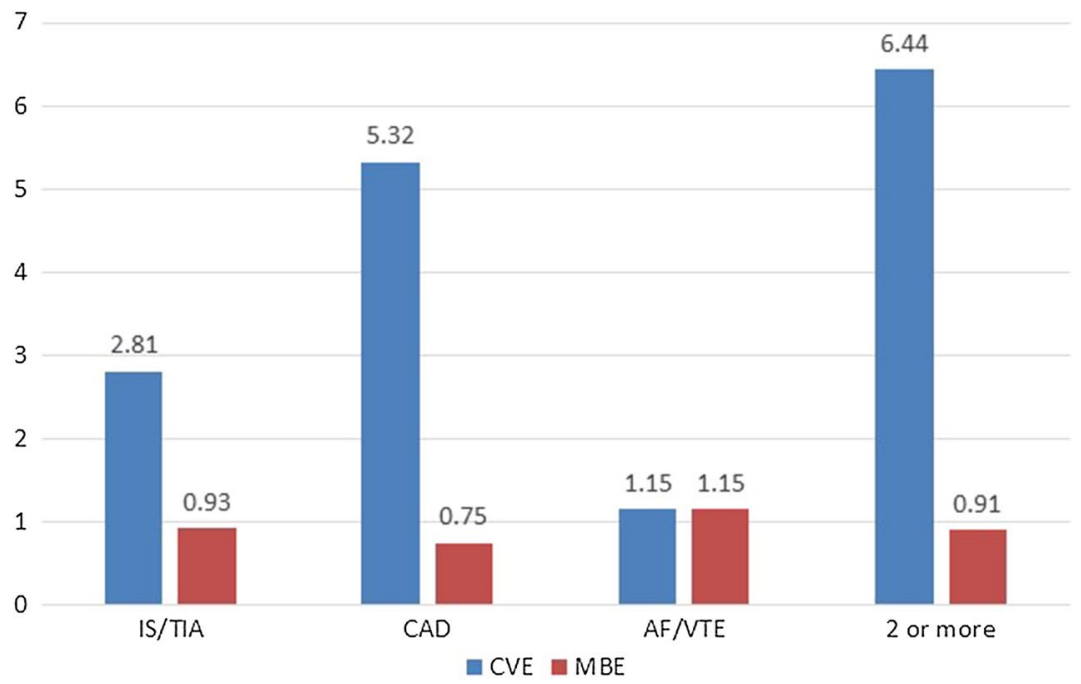

IS; ischemic stroke, TIA; transient ischemic attack, CAD; coronary artery disease, AF; atrial fibrillation, VTE; venous thromboembolism, CVE; cardiovascular events, MBI; major bleeding events
7801 patients with clinically evident CV disease or multiple risk factors [11]. In a subanalysis of CHRISMA on bleeding complications, severe bleeding was observed in 102 patients (1.3\%) who were receiving aspirin alone [12].

The lower risk of CV events in the MAGIC study may suggest the recent progress of risk factor management. However, the risk of CV events was still high enough for aspirin use in our patients with IS/TIA and CAD, on the basis of the risk-benefit balance, as shown in Fig. 2 [5-7]. On the other hand, the incidence rates of $\mathrm{CV}$ and bleeding events were the same in our patients with AF/VTE, as shown in Fig. 2. Namely, CV risk was lower in the patients with AF/ VTE than in those with IS/TIA, CAD, and multiple diseases, while the bleeding risk in the patients with AF/VTE was comparable with those in the other patient categories. Therefore, our results suggested that aspirin is not recommended for the prevention of $\mathrm{CV}$ events in patients with AF/VTE.

Most AF patients recruited to the MAGIC study were unsuitable or unwilling to receive warfarin or at a low risk of stroke. According to the results of the AVERROES (Apixaban Versus Acetylsalicylic Acid [ASA] to Prevent Stroke in Atrial Fibrillation Patients Who Have Failed or Are Unsuitable for Vitamin K Antagonist Treatment), stroke or systemic embolism occurred more frequently in the aspirin group than in the apixaban group, and major bleeding occurred equally in both treatment groups among patients unsuitable to receive vitamin $\mathrm{K}$ antagonists and at risk for stroke [13, 14]. Therefore, aspirin showed no potential for stroke prevention in AF patients. In reality, guidelines of the European Society of Cardiology do not recommend aspirin use under any condition for stroke prevention in AF patients [15]. With aspirin, CV events are difficult to prevent in patients with VTE. According to the Cochrane Database Systematic Review, aspirin did not reduce recurrent VTE, VTE-related mortality, stroke, or MI as compared with placebo for extended prophylaxis in patients with unprovoked VTE [16].

Aspirin is associated with risk of GI toxicity, leading to ulceration and bleeding. For patients taking aspirin, who are at risk of GI events, concomitant use of proton pump inhibitor (PPI) is currently recommended. However, PPI is under-prescribed in these patients [17]. In the MAGIC study, patients receiving PPI had lower risk of GI ulcer or erosion [9]. Therefore, PPI should be more widely used concomitantly with aspirin to reduce GI toxicity for long-term prevention of $\mathrm{CV}$ events in patients with history of $\mathrm{CV}$ diseases.

As study limitations, systematic screening was not conducted in each hospital for patient recruitment. Our registry recruited patients receiving low-dose aspirin who were at high risk of $\mathrm{CV}$ events in the routine clinical practice of selected sites. Inclusion bias may be a potential limitation of this study. In addition, the MAGIC study was a prospective observational registry, but the events were judged by investigators at individual sites and not by a central Event Adjudication Committee, which was not set up in this study. Obviously, our study is not a randomized controlled trial. Although we have a monitoring committee overseeing our registry, the quality of our registry data is not in compliance with GCP.

\section{Conclusions}

Despite various limitations, we believe that the MAGIC study prospectively suggests the risk of $\mathrm{CV}$ and major bleeding events at 1-year follow-up in patients with a history of CV disease who were given aspirin. The results of the MAGIC study may provide important data for future clinical trials and may be refined by a future high-quality database. 
Acknowledgements We would like to greatly thank for the cooperation of members of the Endoscopic Evaluation Committee members; Takashi Kawai MD, PhD, Professor, Department of Gastroenterological endoscopy, Tokyo Medical University, Shinichi Nakamura MD, Professor, Department of Gastroenterology, Tokyo Women's Medical University, Choitsu Sakamoto MD, Professor Emeritus Department of Gastroenterology, Nippon Medical School, Graduate School of Medicine, and Hidekazu Suzuki MD, PhD, Professor, Medical Education Center, Keio University School of Medicine.

A complete list of the Management of Aspirin-induced Gastrointestinal Complications (MAGIC) Study Group

The Organization committee includes Yasuo Ikeda (Chair), Shinichiro Uchiyama, Yasushi Okada, Kazuyuki Shimada, Shinya Goto, Kentaro Sugano, Hideyuki Hiraishi, Naomi Uemura, and Hideki Origasa. The Endoscopic Evaluation Committee includes Naomi Uemura (Chair), Takashi Kawai, Shinichi Nakamura, Chouitsu Sakamoto, and Hidekazu Suzuki. The Event ascertainment committee includes Shinichiro Uchiyama (Chair), Yasushi Okada, Kazuyuki Shimada, and Shinya Goto. The Data Monitoring Committee includes Saichi Hosoda (Chair), Yukito Shinohara, and Toshifumi Hibi. The Data coordinating center includes Hiroko Usami.

List of participating investigators: (Institute, number of enrollment), Department, and investigators' name

(National Center for Global Health and Medicine, 278) Gastroenterology Junichi Akiyama, Naomi Uemura Cardiology Michiaki Hiroe, Osamu Okazaki (Hiroshima University Hospital School of Medicine, 70) Gastroenterology Rie Hanaoka, Hiroki Imagawa, Shinobu Imagawa, Masanori Ito, Shosuke Kitamura, Takayasu Kuwahara, Taiji Matsuo, Sayaka Oba, Toshiko Onitake, Youji Sanomura, Takayoshi Shisido, Akemi Takamura, Shinji Tanaka, Masana Tatsugami, Yoshihiro Wada, Shigeto Yoshida Neurology Masayasu Matsumoto, Toshiho Ohtsuki (Tokai University School of Medicine, 67) Gastroenterology Jun Koike, Masashi Matsushima, Tetsuya Mine, Takayuki Shirai, Takayoshi Suzuki, Kenichi Watanabe Cardiology Shinya Goto, Teruhisa Tanabe, Koichiro Yoshioka Neurology Shigeharu Takagi Neurosurgery Mitsunori Matsumae (Tokyo Medical University Ibaraki Medical Center, 62) Gastroenterology Tsuyoshi Hirayama, Tadashi Ikegami, Shinichi Ito Junichi Iwamoto Cardiology Masamitsu Asano, Akihiro Fukuda, Shinji Okubo Neurology Kaoru Yamazaki (Dokkyo Medical University, 59) Gastroenterology Hideyuki Hiraishi, Takafumi Hoshino, Jun Ishikawa, Kazunari Kanke, Mitsunori Maeda, Rieko Ogura, Yutaka Okamoto, Yasuyuki Saifuku, Takako Sasai, Makoto Suzuki, Akihiro Tajima, Keiichi Tominaga, Hidetaka Watanabe, Michiko Yamagata Cardiology Shigeo Horinaka, Toshihiko Isimitsu, Kimihiko Ishimura, Koichi Kono, Hiroaki Matsuoka, Akihisa Yabe, Hiroshi Yagi Neurology Yasuhisa Daimon, Atsuko Ebata, Koichi Hirata, Hidehiro Takekawa (Uji Hospital, 57) Gastroenterology Tadashi Higaki, Kennji Mayumi, Nami Takeda Cardiology Shohei Sawada, Kaoru Shirai (Tohoku University School of Medicine, 55) Gastroenterology Katsunori Iijima, Shuichi Ohara Cardiology Hiroaki Shimokawa, Morihiko Takeda (Keio University School of Medicine, 53) Gastroenterology Rieko Bessho, Norifumi Hibi, Naoki Hosoe, Yousuke Ida, Hiroyuki Imaeda, Nagamu Inoue, Eisuke Iwasaki, Juntaro Matsuzaki, Hiromasa Nakamizo, Haruhiko Ogata, Yoshimasa Saito, Hidekazu Suzuki Cardiology Akio Kawamura, Daihiko Hakuno, Mitsushige Murata, Satoshi Ogawa, Motoaki Sano Neurology Masayuki Ohira, Shigeaki Suzuki, Toshihiko Shimizu, Norihiro Suzuki, Shinichi Takahashi, Yutaka Tomita (Hamamatsu University School of Medicine, 45) Clinical Research Development Center Takahisa Furuta, Mutsuhiro Ikuma, Masafumi Nishino Neurosurgery Hisaya Hiramatsu, Hiroki Namba Clinical pharmacology Kazuhiko Takeuti, Kazuo Umemura, Akiko Utsumi, Hiroshi Watanabe (Teine Keijinkai Hospital, 40) Gastroenterology Mitsuharu Fukasawa, Akio Katanuma, Toshifumi Kin, Fukuo Komaba, Akira Kurita, Takeshi Matsui, Shinya Mitsui, Hiroyuki Nishimori, Masafumi Nomura, Maki Ohtsubo, Manabu Osanai, Hayato Shida, Kuniyuki Takahashi, Kei Yane Cardiology Harutatsu Muto, Shigemichi Tanaka (University of Toyama, Faculty of Medicine, 39) 3rd Dept. Internal Medicine Ayumu Hosokawa, Toshihiko Kudo, Takako Miyazaki, Tadahiro Orihara, Toshiro Sugiyama, 2nd Dept. Internal Medicine Tadakazu Hirai, Hiroshi Inoue, Tomoki Kameyama, Akira Matsuki, Takashi Nozawa Neurology Yoshiharu Taguchi, Shutaro Takashima, Kortaro Tanaka Neurosurgery Shunro Endo, Hideo Hamada, Nakamasa Hayashi, Naoya Kuwayama (Oji General Hospital, 39) Gastroenterology Tadashi Doi, Akihito Fujimi, Yuji Kanisawa, Hidetoshi Ota, Toshinori Okuda, Yasuhiro Sato Cardiology Katsuhisa Ishii, Nobuo Kato, Tomoaki Matsumoto, Hitoshi Ooiwa, Daisuke Yoshida Neurosurgery Yoshifumi Horita, Shigeki Kashiwabara, Takeshi Mikami (Fujita Health University, 35) Gastroenterology Ichiro Hirata, Tomoyuki Shibata Cardiology Masatsugu Iwase, Yukio Ozaki, Masayoshi Sarai, Eiichi Watanabe Neurology Kunihiko Asakura, Takateru Mihara, Tatsuro Mutoh, Takako Takeuchi, Akihiro Ueda, Madoka Ueda (Gunma University School of Medicine, 34) Endoscopy and Endoscopic Surgery Hiroko Hosaka, Osamu Kawamura, Motoyasu Kusano, Yasuyuki Shimoyama, Cardiology Masashi Arai, Yoshiaki Kaneko, Masahiko Kurabayashi, Akihiko Nakano Neurology Koichi Okamoto (Kokura Memorial Hospital, 32) Gastroenterology Tomoharu Yoshida Cardiology Yoshio Kazuno (Odate Municipal General Hospital, 27) Gastroenterology Hitoshi Ogasawara Neurosurgery Masahiro Sasaki, Kenichi Shibata (Nakamura Memorial Hospital, 25) Neurosurgery Jyoji Nakagawara, Yoshinobu Seo, Toshiiti Watanabe (Iwate Medical University, 25) Gastroenterology Toshimi Chiba, Kazuyuki Suzuki Neurology and geriatric Kuniko Watanabe, Hisashi Yonezawa Yasuo Terayama (Chiba University School of Medicine, 24) Gastroenterology Hitoshi Maruyama, Osamu Yokosuka Cardiology Hiroshi Akazawa, Michiko Daimon, Hiroshi Hasegawa, Naoki Ishio, Issei Komuro, Nakabumi Kuroda, Yoichi Kuwabara, Hideyuki Miyauchi, Taichi Murayama, Toshio Nagai, Keiichi Nakagawa, Tohru Oka, Satoshi Shindo, Ichiro Shiojima, Hiroyuki Takano, Haruhiro Toko, Osamu Yokosuka (Institute of Brain and Blood Vessels Mihara Memorial Hospital, 22) Gastroenterology Takayuki Takahashi Neurology Ban Mihara, Yutaka Tomita (Tokyo Women's Medical University, 22) Gastroenterology Hiromi Haruyama, Maiko Kishino, Hiroyuki Konishi, Shinichi Nakamura, Keiko Shiratori Cardiology Nobuhisa Hagiwara, Kagari Murasaki, Yoshio Uetsuka Neurology Tomomi Kimura, Kenji Maruyama, Yoko Masuda, Sono Tooi, Shinichiro Uchiyama (Nanpu Hospital, 21) Gastroenterology Takako Imamura, Hiromitsu Torimaru, Akio Matsuda, Tooru Niihara, Tatsuyuki Nioh, Hiroto Nishimata, Syunji Shimaoka, Kotaro Tashiro Cardiology Kazuaki Kiyonaga, Shinichirou Toyoshima Neurosurgery Kazuhiro Kusumoto, Shunichi Yokoyama (Sapporo Medical University, 21) 1st Dept. Internal Medicine Yoshiaki Arimura, Akira Goto, Masayo Hosokawa, Hiroyuki Okuda, Yasuhisa Shinomura, Tokuma Tanuma, Hiroyuki Yamamoto, Kentaro Yamashita 2nd Dept. Internal Medicine Akiyoshi Hashimoto, Kazuaki Shimamoto, Nobuhiko Togashi, Kazufumi Tsuchihashi Neurosurgery Kiyohiro Hokin, Masaki Saitoh (Kamiiida Daiichi General Hospital, 20) Gastroenterology Kosuke Tachi Cardiology Satoshi Isobe (Nippon Medical University, 20) Gastroenterology Kazumasa Miyake, Choitsu Sakamoto Cardiology Kyoichi Mizuno, Hitoshi Takano (Jichi Medical University, 20) Gastroenterology Hironari Ajibe, Tomosuke Hirasawa, Hiroyuki Osawa, Kiichi Satoh, Kentaro Sugano, Sachiko Toma, Toru Yoshida Cardiology Yukihiro Hojo, Satoshi Hoshide, Mitsunobu Murata, Kazuyuki Shimada, Masahisa Shimpo, Nozomu Takahashi, Shuichi Ueno, Keiji Yamamoto (Nayoro City General Hospital, 20) Gastroenterology Hiroki Saito, Neurosurgery Kazuhiro Sako (Hakodate Goryokaku Hospital, 19) Gastroenterology Kaoru Kasahara, Toshihisa Kobayashi, Nobuaki Sugawara, Ryo Suzuki, Hiroyuki Takamaru, Hidenori Yamauchi, Atsushi Yawata Cardiology Hiroshi Oimatsu (Nihon University School of Medicine Itabashi Hospital, 18) Gastroenterology and hepatology Shigeaki Mizuno, Mitsuhiko Moriyama Cardiology Masaaki Chiku, Atsushi Hirayama, Junko Honye, Satoshi Kunimoto (Ichinomiya Municipal Hospital, 18) Gastroenterology Chiyuki Chujyou, Youichi Iguchi, Shinichi Kanamori, Keiji Mizutani, Arihiro Nakano, Toshihiro Yamanaka Cardiology Masako Oosawa, Tetsuo Shibata, Michiharu Yamada (Kawasaki 
Medical School, 17) Gastroenterology Ken Haruma, Akiko Shiotani Cardiology Yoji Neishi, Kiyoshi Yoshida (NTT Medical Center Tokyo, 17) Gastroenterology Nobuyuki Matsuhashi Cardiovascular Surgery Osamu Tagusari Stroke Unit Yumiko Yamaoka (Saitama Medical Center, 16) Gastroenterology and hepatology Katsuya Chinen, Shuko Isida, Kazuhito Kani, Keiko Satou, Koji Yakabi Neurology Kyoichi Nomura (Saiseikai Kumamoto Hospital, 15) Gastroenterology Masayoshi Uehara, Shuji Tada Cardiology Junjiroh Koyama, Koichi Nakao Neurology Toshiro Yonehara (Mie University Faculty of Medicine, 15) Endoscopy and Endoscopic Surgery Noriyuki Horiki, Ichiro Imoto, Kyosuke Tanaka Cardiology Masaaki Ito, Hiroshi Nakashima, Muneyoshi Tanimura, Akihiro Tsuji Neurology Akira Taniguchi, Hidekazu Tomimoto Clinical Research Support Center Masakatsu Nishikawa (Sapporo City General Hospital, 13) Gastroenterology Michio Nakamura, Shuji Nishikawa Cardiology Hiroyuki Fukuda, Noriyoshi Kato Neurosurgery Masayoshi Takigami (Yokohama City University Medical Center, 12) Gastroenterology Atsushi Kokawa, Katsuaki Tanaka Cardiovascular Center Kiyoshi Hibi, Kazuo Kimura, Kengo Tsukahara (National Hospital Organization Kagoshima Medical Center, 12) Gastroenterology Hiromitu Fujisima, Naoko Tsubouchi, Cerebrovascular diseases Rikuzo Hamada (Hokkaido University School of Medicine, 11) Gastroenterology Masahiro Asaka, Mototsugu Kato, Shouko Ono Cardiology Tomoo Furumoto, Daisuke Gotou, Naoki Ishimori, Nozomu Kawashima, Mamoru Sakakibara, Takamitsu Souma, Hiroyuki Tsutsui (Yokohama Sakae Kyosai Hospital, 11) Gastroenterology Hiroo Satoh Neurosurgery Yoshihisa Kitamura, MotohiroNomura, Hiroshi Shima (Komaki City Hospital, 10) Gastroenterology Akihiro Miyata, Itaru Ohyama Cardiology Hajime Imai, Taizo Kondo (National Hospital Organization Yokohama Medical Center, 9) Gastroenterology Shouzo Matsushima Cardiology Kazunori Iwade (Shimane University Faculty of Medicine, 8) Gastroenterology Yuji Amano, Kenji Furuta, Norihisa Ishimura, Yoshikazu Kinoshita, Kenji Koshino Cardiology Yutaka Ishibashi, Kazuaki Tanabe Neurology Hirokazu Bokura, Hiroaki Oguro, Shuhei Yamaguchi (Oita University Faculty of Medicine, 8) Gastroenterology Toshio Fujioka, Kazunari Murakami, Tadayoshi Okimoto, Jin Tanahashi Cardiology Munenori Kotoku, Shigeru Naono, Takashi Sato, Akira Tamura (National Hospital Organization Kyushu Medical Center, 7) Gastroenterology Naohiko Harada Cerebrovascular diseases Shinji Satoh Neurology Toshiyasu Ogata, Yasushi Okada (Teikyo University Hospital, 6) Gastroenterology Yasushi Kuyama, Takatsugu Yamamoto Cardiology Takaaki Isshiki, Shuichi Ishikawa, Ken Kozuma, Yoshitaka Shiratori, Naoyuki Yokoyama, Hidenori Watanabe (Kushiro City General Hospital, 6) Gastroenterology Kazuhiko Yonezawa Neurosurgery Toshio Imaizumi (Niigata University School of Medicine, 5) Gastroenterology Yutaka Aoyagi, Yuichi Sato Cardiology Yoshifusa Aizawa, Satoru Hirono Neurology Yasuhisa Akaiwa,Masatoyo Nishizawa Neurosurgery Yukihiko Fujii, Kazuhiko Nishino (University of Yamanashi Faculty of Medicine, 4) Gastroenterology Nobuyuki Enomoto, Tadashi Sato Neurosurgery Kazuya Kanemaru, Hiroyuki Kinouchi, Masao Sugita (NTT Medical Center Sapporo, 4) Gastroenterology Nobuhiko Abe, Shigeru Furukawa, Akihito Kobayashi, Tatsumi Koshiyama, Kimitoshi Kubo, Ken Nishi, Amane Oota, Kazuhiro Takahashi, Youko Tsukuda, Akiko Yokoyama Cardiology Tetsuro Kohya, Noriyuki Miyamoto (Konan Kosei Hospital, 4) Gastroenterology Yoji Sasaki Cardiology Shinichi Ishikawa (Ehime University School of Medicine, 4) Gastroenterology Naoyuki Higaki, Yoshiou Ikeda, Hidehiro Murakami Cardiology Jitsuo Higaki, Akiyoshi Ogimoto, Neurosurgery Takanori Ohnishi, Hideaki Watanabe (Jichi Medical Unversity Saitama Medical Center, 4) Gastroenterology Yukio Yoshida Cardiology Kenshiro Arao, Shin-Ichi Momomura, Kenichi Sakakura (Tosei General Hospital, 3) Gastroenterology Takao Hayasi, Tetsuo Matsuura, Keiichi Morita, Toyohiro Sakata, Yuko Simizu Cardiology Takahiro Kannbara, Kazuyoshi Sakai, Yusuke Uemura (Saga Medical School Faculty of Medicine, Saga University, 3) Gastroenterology Kazuma Fujimoto, Yasuhisa Sakata, Ryo Shimoda, Seiji Tsunada Cardiology Shigemasa Hashimoto, Koichi Node, Tadashi Yamamoto (Tsuchiya General Hospital, 3)
Gastroenterology Hirohisa Kai, Seizi Tao Cardiology Yasuhiko Hayashi, Tomoharu Kawase, Takehito Tokuyama (National Hospital Organization Okayama Medical Center, 2) Gastroenterology Yoshihiro Oofuji Cardiology Hiromi Matsubara (National Defense Medical College, 2) Gastroenterology Ryota Hokari, Atsushi Kawaguchi, Soichiro Miura Cardiology Fumitaka Ohsuzu (Toda Chuo General Hospital, 2) Gastroenterology Youji Harada Cardiology Tadashi Nagao, Takashi Uchiyama (Yamaguchi University Faculty of Medicine, 2) Endoscopy and Endoscopic Surgery Shingo Higaki, Jun Nishikawa Cardiology Masunori Matsuzaki, Toshirou Miura (Shinshu University School of Medicine, 2) Endoscopy Taiji Akamatsu Cardiology Uichi Ikeda, Hiroki Kasai (Research Institute for Brain and Blood Vessels-Akita, 1) Gastroenterology Tsuyoshi Mukoujima Neurosurgery Akifumi Suzuki (Suzuran Clinic, 1) Masahide Wad

Author contribution SU wrote the first draft of the manuscript. All authors reviewed and edited the manuscript and approved the final version of the manuscript.

Funding This study was supported by Japan Cardiovascular Research Foundation (Grant No. 176). The sponsor foundation had no role on the study design, selection of study institution, selection of the committee members, data analysis, or the writing of the manuscript.

\section{Compliance with ethical standards}

Conflict of interest SU received honoraria for consultancy and lecture fees and participation in clinical trials from Bayer, Boehringer Ingelheim, Daiichi-Sanyo, Bristol-Myers Squibb, AstraZeneca and Astellas Amgen, and research grant from Japan Cardiovascular Research Foundation. SG received research grants from Sanofi, Ono, Bristol-Myer Squibb and Pfizer. KS received grants from AstraZeneca, Takeda, Astellas and Daiichi-Sankyo and also sat on advisory panels for AstraZeneca, and Takeda. All other authors declare that there is no conflict of interest.

Informed consent Written informed consent was obtained from all subjects before the study.

Ethical approval The study was approved by an institutional review committee at each participating center.

Open Access This article is distributed under the terms of the Creative Commons Attribution 4.0 International License (http://creativeco mmons.org/licenses/by/4.0/), which permits unrestricted use, distribution, and reproduction in any medium, provided you give appropriate credit to the original author(s) and the source, provide a link to the Creative Commons license, and indicate if changes were made.

\section{References}

1. Antithrombotic Trialists' Collaboration (2002) Collaborative meta-analysis of randomised trials of antiplatelet therapy for prevention of death, myocardial infarction, and stroke in high-risk patients. BMJ 324:71-86

2. Antithrombotic Trialists' (ATT) Collaboration (2009) Aspirin in the primary and secondary prevention of vascular disease: collaborative meta-analysis of individual participant data from randomised trials. Lancet 373:1849-1860

3. Weisman SM, Graham DY (2002) Evaluation of the benefits and risks of low-dose aspirin in the secondary prevention of 
cardiovascular and cerebrovascular events. Arch Intern Med 162:2197-2202

4. Patrono C, Garcia Rodriguez LA, Landolfi R, Baigent C (2005) Low-dose aspirin for the prevention of atherothrombosis. N Engl J Med 353:2373-2383

5. Pearson TA, Blair SN, Daniels SR, Eckel RH, Fair JM, Fortmann SP, Franklin BA, Goldstein LB, Greenland P, Grundy SM, Hong Y, Miller NH, Lauer RM, Ockene IS, Sacco RL, Sallis JF Jr, Smith SC Jr, Stone NJ, Taubert KA (2002) AHA Guidelines for Primary Prevention of Cardiovascular Disease and Stroke: 2002 Update: Consensus Panel Guide to Comprehensive Risk Reduction for Adult Patients without Coronary or Other Atherosclerotic Vascular Diseases. American Heart Association Science Advisory and Coordinating Committee. Circulation 106:388-391

6. Hayden M, Pignone M, Phillips C, Mulrow C (2002) Aspirin for the primary prevention of cardiovascular events: a summary of the evidence for the U.S. Preventive Services Task Force. Ann Intern Med 136:161-172

7. DeFilippis AP, Young R, Carrubba CJ, McEvoy JW, Budoff MJ, Blumenthal RS, Kronmal RA, McClelland RL, Nasir K, Blaha MJ (2015) An Analysis of calibration and discrimination among multiple cardiovascular risk scores in a modern multiethnic cohort. Ann Intern Med 162:266-275

8. Origasa H, Goto S, Shimada K, Uchiyama S, Okada Y, Sugano K, Hiraishi H, Uemura N, Ikeda Y, MAGIC Investigators (2011) Prospective cohort study of gastrointestinal complications and vascular diseases in patients taking aspirin: rationale and design of the Magic study. Cardiovasc Drugs Ther 25:551-560

9. Uemura N, Sugano K, Hiraishi H, Shimada K, Goto S, Uchiyama S, Okada Y, Origasa H, Ikeda Y, MAGIC Study Group (2014) Risk factor profiles, drug usages, and prevalence of aspirin-associated gastroduodenal injuries among high-risk cardiovascular Japanese patients: the results from the MAGIC study. J Gastroenterol 49:814-824

10. CAPRIE Steering Committee (1996) A randomised, blinded trial of clopidogrel versus aspirin in patients at risk of ischaemic events (CAPRIE). Lancet 348:1329-1339

11. Bhatt DL, Fox KA, Hacke W, Berger PB, Black HR, Boden WE, Cacoub P, Cohen EA, Creager MA, Easton JD, Flather MD, Haffner SM, Hamm CW, Hankey GJ, Johnston SC, Mak KH, Mas JL, Montalescot G, Pearson TA, Steg PG, Steinhubl SR, Weber MA, Brennan DM, Fabry-Ribaudo L, Booth J, Topol EJ, CHARISMA Investigators (2006) Clopidogrel and aspirin versus aspirin alone for the prevention of atherothrombotic events. N Engl J Med 354:1706-1717

12. Berger PB, Bhatt DL, Fuster V, Steg PG, Fox KA, Shao M, Brennan DM, Hacke W, Montalescot G, Steinhubl SR, Topol EJ, CHARISMA Investigators (2010) Bleeding complications with dual antiplatelet therapy among patients with stable vascular disease or high risk factors for vascular disease: results from the Clopidogrel for High Atherothrombotic Risk and Ischemic Stabilization, Management, and Avoidance (CHARISMA) trial. Circulation 121:2575-2583

13. Connolly SC, Eikelboom J, Joyner C, Diener HC, Hart R, Golitsyn S, Flaker G, Avezum A, Hohnloser SH, Diaz R, Talajic M, Zhu J, Pais P, Budaj A, Parkhomenko A, Jansky P, Commerford P, Tan RS, Sim KH, Lewis BS, Van Mieghem W, Lip GY, Kim JH, Lanas-Zanetti F, Gonzalez-Hermosillo A, Dans AL, Munawar M, O’Donnell M, Lawrence J, Lewis G, Afzal R, Yusuf S, AVERROES Steering Committee Investigators (2011) Apixaban in patients with atrial fibrillation. N Engl J Med 364:806-817

14. Diener HC, Eikelboom J, Connolly SJ, Joyner CD, Hart RG, Lip GY, O’Donnell M, Hohnloser SH, Hankey GJ, Shestakovska O, Yusuf S, AVERROES Steering Committee Investigators (2012) Apixaban versus aspirin in patients with atrial fibrillation and previous stroke or transient ischaemic attack: a predefined subgroup analysis from AVERROES, a randomized trial. Lancet Neurol 11:225-231

15. Kirchhof P, Benussi S, Kotecha D, Ahlsson A, Atar D, Casadei B, Castella M, Diener HC, Heidbuchel H, Hendriks J, Hindricks G, Manolis AS, Oldgren J, Popescu BA, Schotten U, Van Putte B, Vardas P, ESC Scientific Document Group (2016) 2016 ESC Guidelines for the management of atrial fibrillation developed in collaboration with EACTS. Eur Heart J 37:2893-2962

16. Robertson L, Yeoh SE, Ramli A (2017) Secondary prevention of recurrent venous thromboembolism after initial oral anticoagulation therapy in patients with unprovoked venous thromboembolism. Cochrane Database Syst Rev. 12:CD011088

17. Lavie CJ, Howden CW, Scheiman JS, Tursi JT (2017) Upper gastrointestinal toxicity with long-term aspirin therapy: consequences and prevention. Curr Probl Cardiol 42:146-164

Publisher's Note Springer Nature remains neutral with regard to jurisdictional claims in published maps and institutional affiliations.

\section{Affiliations}

\section{Shinichiro Uchiyama ${ }^{1}$ (D) Shinya Goto ${ }^{2} \cdot$ Hideki Origasa $^{3} \cdot$ Naomi Uemura $^{4} \cdot$ Kentaro Sugano $^{5} \cdot$ Hideyuki Hiraishi $^{6,7}$. Kazuyuki Shimada ${ }^{8} \cdot$ Yasushi Okada $^{9} \cdot$ Yasuo Keda $^{10}$ on behalf of The MAGIC Study Group}

1 Clinical Research Center for Medicine, International University of Health and Welfare, Center for Brain and Cerebral Vessels, Sanno Hospital and Sanno Medical Center, 8-5-35 Akasaka, Minato-ku, Tokyo 107-8332, Japan

2 Department of Medicine (Cardiology), Tokai University School of Medicine, Isehara, Kanagawa, Japan

3 Department of Biostatistics and Clinical Epidemiology, University of Toyama School of Medicine, Toyama, Japan

4 Department of Gastroenterology and Hepatology, Kohnodai Hospital, National Center for Global Health and Medicine, Chiba, Japan

5 Department of Medicine, Division of Gastroenterology, Jichi Medical University, Shimotsuke, Tochigi, Japan
6 Department of Gastroenterology, Dokkyo Medical University, Mibu, Tochigi, Japan

7 AOI Nanasawa Rehabilitation Hospital, Atsugi, Kanagawa, Japan

8 Department of Medicine, Division of Cardiology, Jichi Medical University, Shimotsuke, Tochigi, Japan

9 Department of Cerebrovascular Medicine and Neurology, National Hospital Organization Kyushu Medical Center Clinical Research Institute, Fukuoka, Japan

10 Graduate School of Advanced Science and Engineering, Waseda University, Tokyo, Japan 